\title{
Modeling fruit microstructure using an ellipse tessellation algorithm
}

\author{
H.K. Mebatsion ${ }^{1 *}$, P. Verboven ${ }^{1}$, Q.T. Ho ${ }^{1}$, B.E. Verlinden ${ }^{2}$, F. Mendoza ${ }^{1}$, T.A. Nguyen ${ }^{1}$, \\ B.M. Nicolaï ${ }^{1,2}$
}

${ }^{1}$ BIOSYST-MeBioS, Katholieke Universiteit Leuven, W. De Croylaan 42, B-3001 Leuven, Belgium, *E-mail: Hibru.Mebatsion@biw.kuleuven.be

${ }^{2}$ Flanders Centre of Postharvest Technology, W. De Croylaan 42, B-3001 Leuven, Belgium

\begin{abstract}
Modeling plant microstructure is of great interest to food engineers to simulate the behavior of the physical properties (e.g., concerning mass transfer, mechanics) of plant tissues at the cellular level. The generation of geometrical models of microscopic structures is considered a prime requirement to develop microscale models to study and describe these properties. For this end, this paper presents a novel ellipse tessellation algorithm to generate a 2D geometrical model of apple tissue. Ellipses are used to quantify the orientation and aspect ratio of cells on a microscopic image. The cell areas and centroids of each cell are also determined by means of a numerical procedure. These characteristic quantities are then described by means of probability density functions. The model tissue geometry is generated from the ellipses which are truncated when neighboring areas overlap. As a result, a virtual microstructure consisting of truncated ellipses fills up the entire space with the same number of cells as that of microscopic images and with similar area, orientation and aspect ratio distribution. The spatial variability of the geometric characteristics (cell area size, cell shape, cell orientation and tissue porosity) of the virtual cellular structure was also evaluated and compared to that of the microscopic images. Statistical analysis showed that the virtual geometry generated with this approach yields spatially equivalent geometries to that of real fruit microstructures. Compared to the more common algorithm of Voronoi diagrams, ellipse tesselation is superior for generating the microstructure of tissue. The extension of the algorithm to $3 \mathrm{D}$ is straightforward. These representative tissues will be exported into a finite element environment via interfacing codes to perform in silico experiments for estimating gas and moisture diffusivities and investigating their relation with fruit microstructure.
\end{abstract}

Keywords: microscopy; finite element model; mass transfer, multiscale modelling

\section{Introduction}

The physical, biological and transport properties of materials depend on the size, shape, and properties of the microstructure as well as their spatial distribution with in the material matrix (Ghosh et al., 1996). Material stability, transport properties, structural integrity or nutritional quality of food materials are the reflections of the assemblies of microstructures, when embedded in, give rise to an engineered structure, which we call macrostructure (tissue and organ) (Aguilera, 2005). The ultimate task becomes the determination of parameters in the leading transport equations on the macroscale given the known structure and heterogeneity of processes at the microstructure (Lammertyn et al., 2001; Lammertyn et al., 2003; Veraverbeke et al., 2003 (1); Veraverbeke et al., 2003 (2); Nguyen et al., 2004; Nguyen et al., 2005; Ho et al., 2005). The transition from the microstructure to macrostructure is then 
achieved by appropriate homogenization procedures. Because fruit tissue is composed of a very large number of cells, its properties depend not only on the characteristics of individual cells but also on the connectivity and interactions among cellular components. Statistical description and modeling of microstructures are an essential means to implement such an approach. During the past decades, several types of Voronoi based models have been used to represent microstructures of different materials such as aggregates of grains in polycrystals (Nygards and Gudmundson, 2002), composite materials (Ingraffea et al., 2002), ceramic microstructures (Espinosa and Zavattieri 2000) and fruit microstructure (Mebatsion et al., 2006). The Poisson Voronoi diagrams (PVDs) have been extensively used and studied by different authors (Mattea et al., 1989; Roudot et al., 1990; Mebatsion et al., 2006). Mebatsion et al. (2006) generated Poisson Voronoi diagrams having similar statistical properties (area, orientation and aspect ratio) as that of apple parenchyma microstructures. However, the spatial statistics of the real cells and the representative PVDs were not evaluated.

Autocorrelation statistics are basic descriptive statistics for any data ordered in space as they provide basic information about the ordering of the data that is not available from other statistical measures such as mean and standard deviation of variables. Spatial autocorrelation statistics detect the presence of interdependence between data at neighboring locations and derive the effect upon the values of the measurements. In other words, spatial autocorrelation statistics measure the amount that the measurements depart from the requirements of independence (Odland, 1988); it may happen that values of a variable sampled at nearby locations are more similar than those sampled at locations more distant from each other. Spatial autocorrelation can occur at multiple spatial scales or vary with spatial orientation. For anisotropic materials, the spatial variability along the $\mathrm{x}$-axis differs from that of the $\mathrm{y}$-axis.

The aim of the presented work was to incorporate spatial statistics properties in to a virtual tissue algorithm. In this respect, this paper presents a novel approach to the generation of statistically and spatially equivalent virtual fruit microstructure using an ellipse tessellation algorithm.

\section{Materials and methods}

\section{Microscopic images geometrical parameter calculation}

The study was based on images of fruit cellular structures obtained from light microscopy. Sample preparation and image acquisition procedures outlined by Mebatsion et al., (2006) were followed. Samples from apple ( $c v$. Cameo) parenchyma tissues were used. The geometrical characteristics of cells were estimated after the transformation of the digital images to representative polygons defined by points on the natural boundary of the cells. From the resulting coordinates, the centroidal points, areas, perimeters, aspect ratios and orientations of each approximated polygonal cells were determined using moment calculation and moment based ellipse-fitting algorithms (Mebatsion et al., 2006).

\section{Virtual tissue generation}

\section{Ellipse tessellation}

Previous studies suggest that microscopic fruit cell sections are elliptically shaped (aspect ratio greater than one) (Mebatsion et al., 2006; Schotsmans, 2003). The ellipse-fitting algorithm approximates every cell of the microstructure with the corresponding ellipse that 
has the same second moment of area, from which aspect ratio and orientation of cellular images is determined.

An ellipse is a conic that can be described by an implicit second order polynomial:

$$
F(x, y)=a x^{2}+b x y+c y^{2}+d x+e y+f=0
$$

with an ellipse-specific constraint:

$$
b^{2}-4 a c<0
$$

where $a, b, c, d, e, f$ are the coefficients of the ellipse and $(x, y)$ are coordinates of sample points lying on it. The polynomial $\mathrm{F}(\mathrm{x}, \mathrm{y})$ is called the "algebraic distance" of a point $(x, y)$ to the conic $F(x, y)=0$. By introducing vectors

$$
\begin{aligned}
& \mathbf{a}=[a, b, c, d, e, f]^{T} \\
& \mathbf{x}=\left[x^{2}, x y, y^{2}, x, y, 1\right]^{T}
\end{aligned}
$$

the above equation can be rewritten as:

$$
F(\mathbf{a} ; \mathbf{x})=\mathbf{a}\lceil\mathbf{x}=\mathbf{0}
$$

Fitting an ellipse to a general conic is to minimize the algebraic distance over the set of data points in the least square sense. To ensure an ellipse-specificity of the solution, because the vector a can be arbitrary scaled, the following constraint equation can be considered instead of equation 2 (Fitzgibbon et al., 1999):

$$
4 a c-b^{2}=1
$$

This is a quadratic constraint, which may be expressed in matrix form $\mathbf{a}^{T} \mathbf{C a}=\mathbf{1}$ as

$$
\mathbf{a}^{T}\left[\begin{array}{rrrrrr}
0 & 0 & 2 & 0 & 0 & 0 \\
0 & -1 & 0 & 0 & 0 & 0 \\
2 & 0 & 0 & 0 & 0 & 0 \\
0 & 0 & 0 & 0 & 0 & 0 \\
0 & 0 & 0 & 0 & 0 & 0 \\
0 & 0 & 0 & 0 & 0 & 0
\end{array}\right] \mathbf{a}=\mathbf{1}
$$

The minimization procedure can be carried out by defining design vector $\chi_{i}$ for the individual points $\left(x_{i}, y_{i}\right)$ as

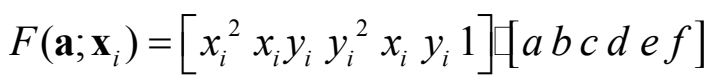

$$
\begin{aligned}
& =\chi_{i} \bar{a}
\end{aligned}
$$


Then assembling the design vectors in to a design matrix $\mathbf{D}$ of size $N \times 6$

$$
\mathbf{D}=\left[\begin{array}{lccccc}
x_{1}^{2} & x_{1} y_{1} & y_{1}^{2} & x_{1} & y_{1} & 1 \\
\vdots & \vdots & \vdots & \vdots & \vdots & \vdots \\
x_{i}^{2} & x_{i} y_{i} & y_{i}^{2} & x_{i} & y_{i} & 1 \\
\vdots & \vdots & \vdots & \vdots & \vdots & \vdots \\
x_{N}{ }^{2} & x_{N} y_{N} & y_{N}{ }^{2} & x_{N} & y_{N} & 1
\end{array}\right]
$$

Following conic sections fitting to scattered data procedure (Fitzgibbon, 1997) the constraint ellipse-fitting problem reduces to

Minimizing $E=\|\mathbf{D a}\|^{2}$, subject to the constraint

$$
\mathbf{a}^{T} \mathbf{C a}=\mathbf{1}
$$

Introducing Lagrange multiplier $\lambda$ and differentiating the following systems of simultaneous equations are obtained (for details refer to Fitzgibbon, 1997).

$$
\begin{aligned}
& \mathbf{D}^{T} \mathbf{D a}-\lambda \mathbf{C a}=\mathbf{0} \\
& \mathbf{a}^{T} \mathbf{C a}=\mathbf{1}
\end{aligned}
$$

The solution of the minimization problem represents the best-fit ellipse for the given set of points.

These ellipses were used to quantify the orientation and aspect ratio of cells on a microscopic image. The cell areas and centroids of each cell were also determined by means of a numerical procedure. The model tissue geometry was generated from the ellipses, which were truncated when neighboring areas overlap. By doing so, as many truncated ellipses as there are cellular images were generated filling the entire cellular space. Fig. 1 shows the mechanism of generating non-overlapping truncated ellipses. For every microscopic cell, an ellipse is fitted and for every fitted ellipse, the algorithm searches a region that is not in the intersection with the rest fitted elliptical regions. This yields a set of truncated ellipses, representing the fruit microstructure. 


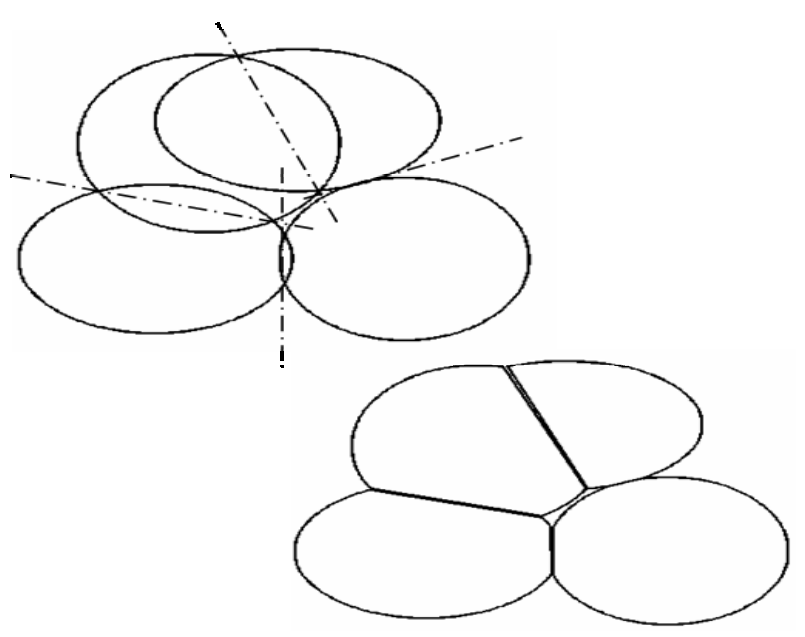

Figure 1: The ellipsoidal virtual tissue generating mechanism.

\section{Statistics}

\section{Statistical distributions}

Fitting distributions to data sets by assuming a pre-specified distribution (e.g. normal distribution) with distribution parameters (e.g. mean and variance) obtained from the data were carried out. Both the assumed and the empirical cumulative distribution functions (CDFs) at each data point were obtained. If the maximum distance between the assumed CDF and the empirical CDF were small, the assumed CDF was accepted as good fit (Justel et al., 1997). In this study, the Kolmogorov-Smirnov test (K-S test) of Goodness of Fit (GoF) at the $5 \%$ level of significance was used.

\section{Spatial Statistics}

In order to calculate spatial statistics, it is necessary to express the spatial arrangement in terms of some function that assigns values to pairs of locations in order to represent their location with respect to one another by means of a "weighting function" (Odland, 1988; Brown, 1982; Cliff and Ord, 1972; Kelejian and Prucha, 2001).

For data sets that are not arranged regularly or represent area units of different size and shape, weights could be defined as distance based weights (Schabenberger and Pierce, 2002; Brown, 1982). In this study, the weighting function was defined as the inverse of the distance between the geometric centres (centroids) of two cellular regions. The two well known local spatial correlation tests namely; Moran's I and Geary's C tests were used to test the spatial variability of the microscopic image and its virtual counterpart.

\section{Moran's I test}

Moran's I test is the most popular test for spatial correlation. The test procedure standardizes the variables by subtracting the mean and deflating by a specific factor (usually the variance). The Moran's I is defined as: 


$$
I=\frac{n}{\sum_{i=1}^{n} \sum_{j=1}^{n} w_{i j}} \frac{\sum_{i=1}^{n} \sum_{j=1}^{n} u_{i} u_{j}}{\sum_{i=1}^{n} u_{i}^{2}} \quad, i \neq j
$$

where $u_{i}=u(i)-E(u), E(u)$ is the expectation value of $u, n$ is the total number of regions in the domain, $u$ is the variable of interest whose correlation statistics is to be measured and $w_{i j}$, is the inverse of the separation of geometrical centres of two regions. In our study, the term $u(i)$ represents the area of the individual cells.

Moran's $I$ is merely the spatial auto covariance, standardized by the variance of the data sets and the weighting function. The weighting function defines the arrangement of regions on a map. It is the measure of connectivity of the set of regions

Moran's $I$ has an expected value of $E(I)=-\left(\frac{1}{n-1}\right)$. Values of $I$ that exceed the expected value indicate positive spatial autocorrelation in which values tend to be similar to neighboring values. Values of $I$ below the expectation indicate negative spatial autocorrelation in which neighboring values tend to be dissimilar. If $I=0$, then $u_{i}$ and $u_{j}$ are independent.

The hypothesis of no spatial autocorrelation is rejected at $\alpha$ (usually $5 \%$ ) significance level if

$$
Z(I)=\frac{|I-E(I)|}{\sigma_{I}}
$$

is more extreme than the $Z_{\alpha / 2}$ cut-off of a standard Gaussian distribution (Cliff and Ord ,1970; Cliff and Ord, 1981; Brown, 1982; Odland, 1988; Schabenberger and Pierce, 2002). $\sigma_{I}$ is the estimate of the standard deviation.

$\sigma_{I}=\left[\frac{n^{2} S_{1}-n S_{2}+3 S_{0}{ }^{2}}{S_{0}^{2}\left(n^{2}-1\right)}\right]^{\frac{1}{2}}$

where $S_{0}=\sum_{i=1}^{n} \sum_{j=1}^{n} w_{i j}, S_{1}=2 \sum_{i=1}^{n} \sum_{j=1}^{n}\left(w_{i j}\right)^{2}$ and $S_{2}=4 \sum_{i=1}^{n}\left(\sum_{j=1}^{n} w_{i j}\right)^{2}$

The p-value, the significance of the statistical tests, can be calculated from the standard normal cumulative probability table using the calculated $\mathrm{Z}$ value as:

$p-$ value $=\operatorname{prob}(Z \geq Z(I))$

When the global statistics of spatial association is not significant, one can proceed to test the individual local spatial association coefficients by the method described in the next section. 


\section{Local indicators of spatial association (LISA)}

The idea of local indicators of spatial association (LISA) is that, although there may be no spatial autocorrelation globally, there might be local pockets of positive or negative spatial autocorrelation in the data, so-called hot spots and cold spots, respectively (Schabenberger and Pierce, 2000; Sokal et al., 1998). Spot definitions based on autocorrelation measures designate sites as unusual if the spatial dependency is locally much different from the other sites. The LISA for each observation also gives an indication of the extent of significant spatial clustering of similar values around an observation. The LISA permits the decomposition of the global coefficient of spatial association into separate parts, making it possible to identify individual locations that are major contributors of the global association [Sokal et al., 1998).The LISA version of the Moran's I is

$$
I_{i}=\frac{n}{\sum_{i}^{n} u_{i}^{2}} u_{i} \sum_{j}^{n} w_{i j} u_{j}
$$

The term $\sum_{j}^{n} w_{i j} u_{j}$ is formally known as the spatial lag for $u$ at location $i$. In the absence of spatial autocorrelation, $I_{i}$ has the expected value $E\left(I_{i}\right)=\frac{-\sum_{i=1}^{n} w_{i j}}{n-1}$. The interpretation of LISA is that if $I_{i}$ is smaller than $E\left(I_{i}\right)$, the sites connected to site $i$ (called pivot site) have attribute values dissimilar from attributes at site $i$. A high (low) value at site $i$ is surrounded by a low (high) values. On the other hand, if $I_{i}$ is greater than $E\left(I_{i}\right)$, sites connected to site $i$ show similar value. A high (low) value at site $i$ is surrounded by a high (low) values. The map of LISA indicates which regions of the domain behave differently from the rest (Sokal et al., 1998).

\section{Geary's C test}

Geary's $C$ test is an alternative statistics for spatial autocorrelation constructed by using another measure of covariation. The test uses the sum of the square difference between pair of data values as a measure of covariation instead of the joint covariance and standardize in a slightly different way to get a correlation defined as follows:

$$
C=\frac{n-1}{2 \sum_{i=1}^{n} \sum_{j=1}^{n} w_{i j}} \frac{\sum_{i=1}^{n} \sum_{j=1}^{n} w_{i j}\left(u_{i}-u_{j}\right)^{2}}{\sum_{i=1}^{n} u_{i}^{2}}
$$

The expectation value of Geary's $C$ is 1 (one) for independence among neighboring values. It is inversely related to Moran's $I$ and it does not provide identical inference as it emphasizes the differences in values between pairs of observations, rather than the covariation between pairs. Moran's I gives more global indicator, whereas the Geary's $C$ coefficient is more sensitive to differences in small neighborhoods. Moreover, the efficiency of Moran's I tests is generally better than that of Geary's C (Odland, 1988). 
The significance of the Geary's $C$ spatial statistics can be calculated in the same way as the Moran's $I$ statistics. The standard deviation under normality assumptions given as (Cliff and Ord, 1972; Cliff and Ord, 1981):

$$
\sigma_{c}=\left[\frac{\left(2 S_{1}+S_{2}\right)(n-1)-4 S_{0}^{2}}{2(n+1) S_{0}^{2}}\right]^{\frac{1}{2}}
$$

All computations were carried out in the Matlab version 7.0.1-programming environment (The Mathworks, Natick, MA).

\section{Results and Discussion}

\section{The geometrical analysis of ellipse tesselation}

Fig. 2 shows an apple microscopic image ( $c v$. Cameo) and its equivalent ellipse tessellation virtual tissue. The shaded regions represent the intercellular regions. The statistical comparison between area, aspect ratio and orientation distributions of the microscopic images and virtual tissues are represented in Fig. 3a-3c. There was a good agreement between all the geometrical property distributions investigated.

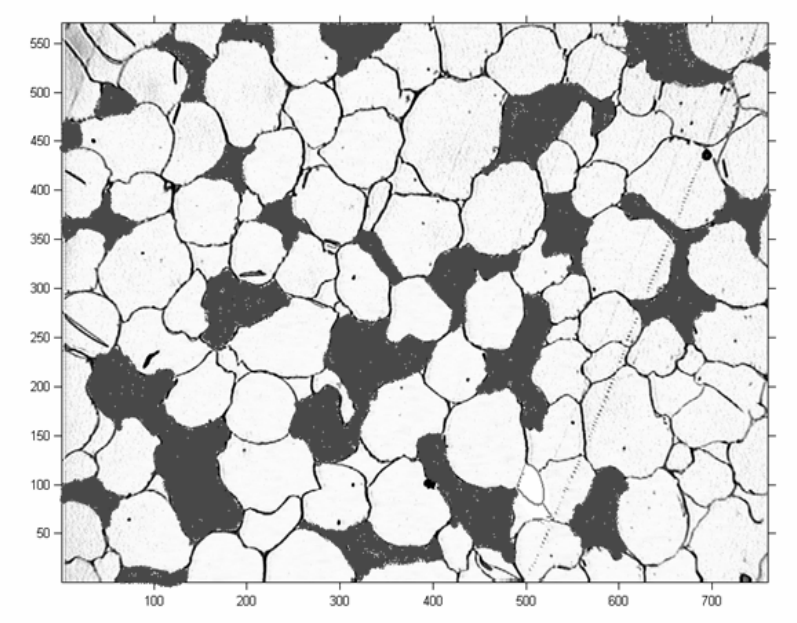

(a) 


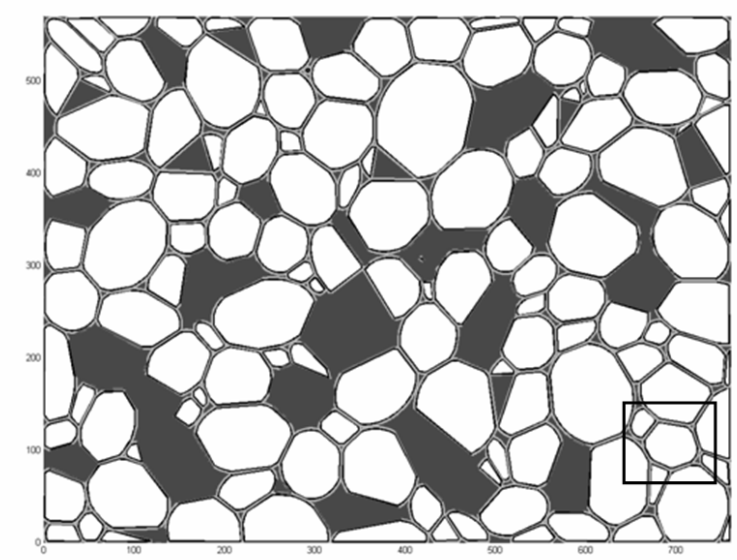

(b)

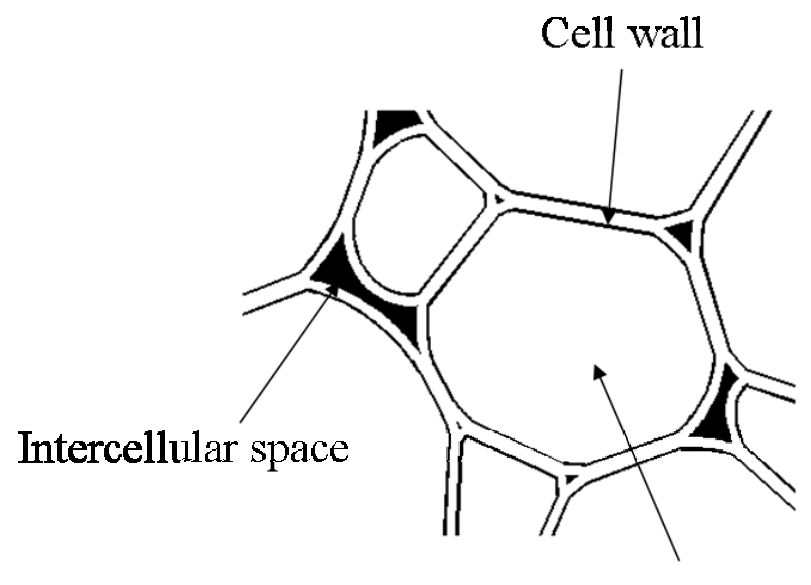

Cell

(c)

Figure 2: Micrograph and ellipse tessellated virtual apple parenchyma tissue: (a) micrograph; (b) virtual tissue; (c) magnified view of virtual tissue. (Dimensions in pixels; 1 pixel $=1.85185 \mu \mathrm{m})$. The dark regions represent intercellular spaces.

Geometrically speaking, all of the results suggest that the ellipse tessellated virtual is a good representative of the micrograph. 
(a)

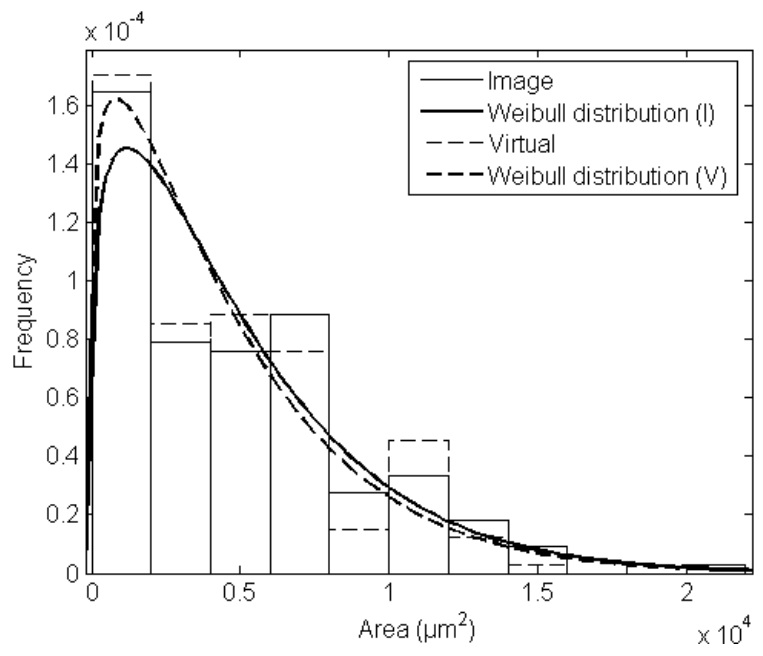

(b)
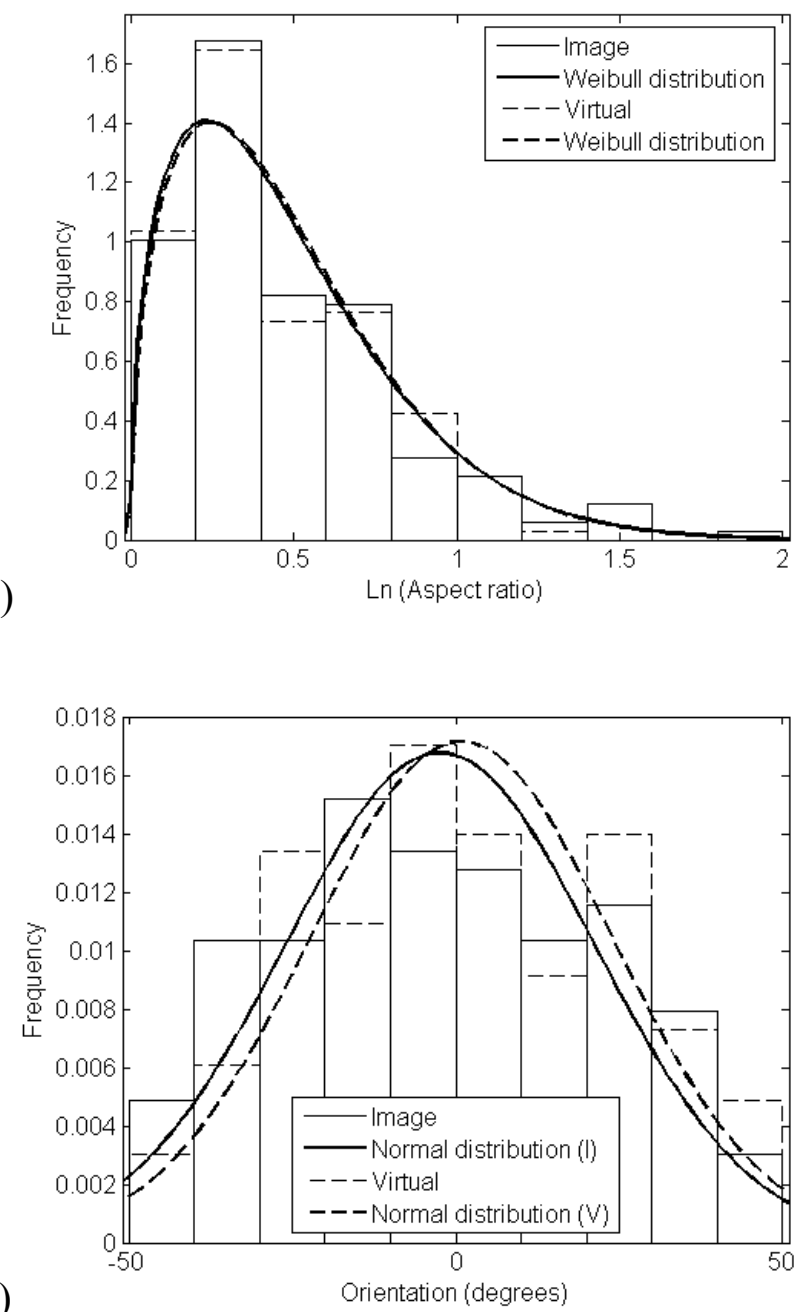

Figure 3: Geometrical properties of cellular images and virtual apple parenchyma tissue: (a) area distribution; (b) aspect ratio distribution; (c) orientation distribution. 


\section{The spatial analysis of ellipse tessellation}

The spatial comparison using Moran's I and Geary's $C$ tests also showed that the virtual tissue was a good approximation of the real microscopic image in the sense of spatial correlation. The Moran's I values of ellipse tessellated virtual tissue is -0.0071 showing negative spatial association. Concerning the $Z$ scale of the ellipse tesselation, there is no enough evidence to reject the null hypothesis of no spatial correlation (with $Z=0.1019<Z_{\alpha / 2}$ or $\mathrm{p}=0.46>0.05$ ). Moreover, the Geary's $C$ value calculated to be equal to 1.0093 supports the presence of spatial similarity between the micrograph (with $C=1.0084$ ) and the ellipse tessellated virtual tissue. The LISA test clearly shows the presence of comparable trend in local spatial associations between microscopic images and ellipse tessellated counterparts (Fig. 4).

(a)

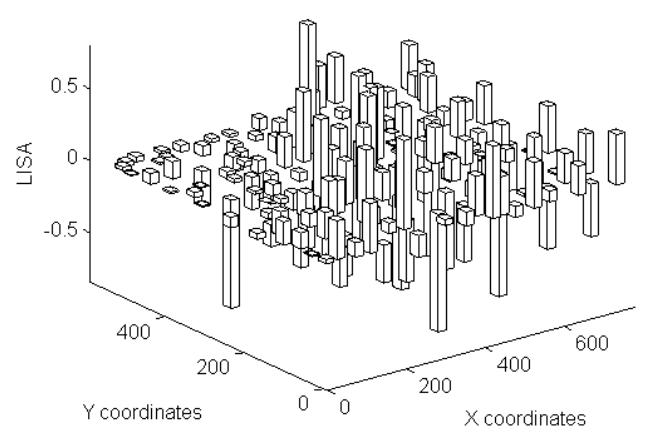

(b)

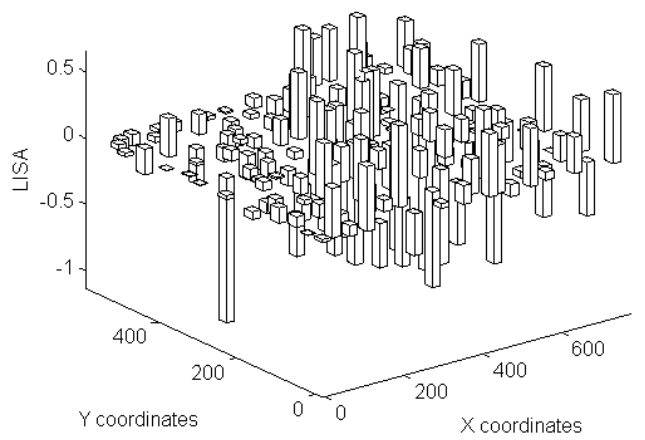

(c)

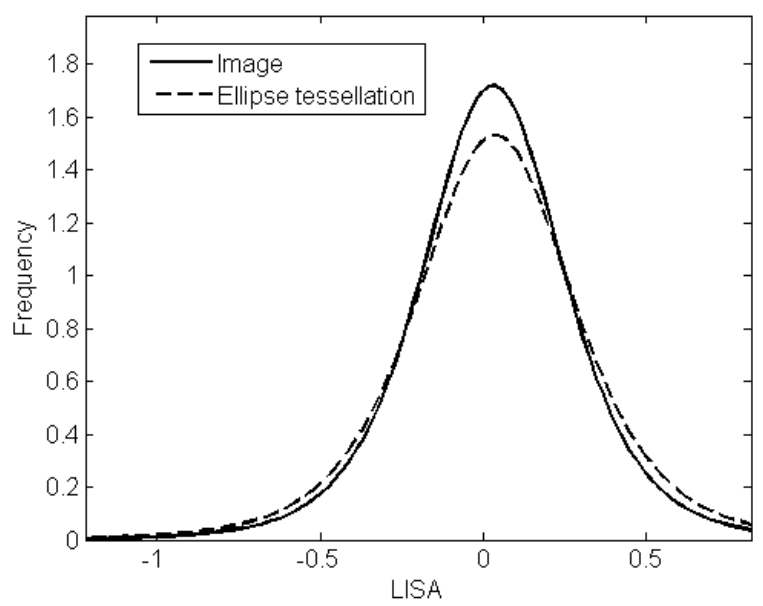

Figure 7: LISA test of the microscopic and ellipse tessellated apple parenchyma tissue: (a) LISA of the micrograph; (b) LISA of Ellipse tessellated virtual tissue; (c) LISA frequency distribution.

\section{Conclusions}

A novel ellipse tesselation algorithm for generation of fruit was developed. Compared to the more common algorithm of Voronoi diagrams, both the centroid based Voronoi diagrams (CVDs) and the Poisson Voronoi diagrams (PVDs), ellipse tesselation was superior for generating the microstructure of tissue. The difficulty of generating Voronoi based virtual tissues for regular microscopic fruit tissues (such as onion) and tissues with large cells surrounded by smaller ones or vice versa were overcome by our new tessellation algorithm. 
The ellipse tessellated virtual tissues were proved spatially and statistically representative to the fruit microstructure.

Such a microstructural model has large potential in multiscale modeling of gas and moisture transport in fruits. It avoids a tiresome classical continuum modeling approach where transfer properties that appear in the macroscopic transport equation are determined experimentally. As such, the heterogeneous properties of tissue and complex microstructural structures are rather recognized and separate model parameters and boundary conditions are defined to perform in silico experiments. This leads to a better understanding of the physics of the process .The transition from microstructure to macrostructure is then achieved by appropriate homogenization procedures.

\section{Acknowledgements}

Financial support by the Flanders Fund for Scientific Research (FWO-Vlaanderen) (project G.0200.02) and the K.U.Leuven (project IDO/00/008 and OT 04/31, IRO PhD scholarship for Q.T. Ho and T.A. Nguyen) are gratefully acknowledged. Pieter Verboven is a postdoctoral researcher of the Flemish Fund for Scientific Research (FWO-Vlaanderen).

\section{References}

Aguilera, J. M. (2005). Why food microstructure? J. Food Eng. 67, 3-11.

Brown, M. A. (1982). Modeling the spatial distribution of suburban crime. Economic Geography. 58(3), 247-261.

Cliff A.D.; Ord K. (1972). Spatial autocorrelation: A review of existing and new measures with applications. Economic Geography. International Geographical Union Proceedings. Commission on Quantitative methods, 46, 269-292.

Cliff, A.D.; Ord, J.K. (1981). Spatial processes: Models \& applications. London, UK.

Espinosa, H.D.; Zavattieri, P. (2000). Modeling of ceramic microstructures: Dynamic damage initiation and evolution. Amer. Inst. Physics. pp 333-338.

Fitzgibbon, A.; Pilu, M.; Fisher, R. B. (1999). Direct least square fitting of ellipses. IEEE Trans. Pattern Anal. Mach. Intel. 21(5), 476-480.

Fitzgibbon, A. (1997). "Stable segmentation of 2D curves" PhD thesis, University of Edinburgh, UK, 125p.

Ghosh, S., Lee, K., Moorthy, S. (1996). Two scale analysis of heterogeneous elastic-plastic materials with asymptotic homogenization and Voronoi cell finite element model. Comput. Meth. Appl.Mech.Eng.132, 63-116.

Ho, Q.T.; Verlinden, B.E.; Verboven, P.; Vandewalle, S.; Nicolaï, B.M. (2005). Simultaneous measurement of oxygen and carbon dioxide diffusivities in pear fruit tissue using optical sensors. J. Sci. Food and Agric. (Accepted)

Ingraffea, A.R.; Iesulauro, E.; Dodhia, K.; Wawrzynek, P.A. (2002). A multiscale modeling approach to crack initiation in aluminium polycrystals. Fifth World Congress on Computational Mechanics, Vienna, Austria.

Justel, A.; Pena, D.; Zamar, R. (1997). A multivariant Kolmogorov-Smirnov test of goodness of fit. Statistics and Probability Letters. 35, 251-259. 
Kelejian, H. H; Prucha, I. R. (2001). On the asymptotic distribution of the Moran I test statistics with applications. Journal of Econometrics. 104, 219-257.

Lammertyn, J.; Scheerlinck, N.; Jancsok, P., Verlinden B.E.; Nicolaï, B.M. (2003). A respiration-diffusion model for 'Conference' pears: model development and validation. Postharvest Biology and Tech. 30, 29-42.

Lammertyn, J.; Scheerlinck, N.; Verlinden, B.E.; Schotsmans, W.; Nicolaï, B.M. (2001). Simultaneous determination of oxygen diffusivity and respiration in pear skin and tissue. Postharvest Biology and Tech. 23, 93-104.

Mattea, M.; Urbicain, M.J.; Rotstein, E. (1989). Computer model of shrinkage and deformation of cellular tissue during dehydration. Chem. Eng. Sci. 44, 2853-2859.

Mebatsion, H. K.; Verboven, P.; Verlinden, B. E.; Ho, Q.T.; Nguyen, T.A.; Nicolaï, B. M. (2006). Microscale modelling of fruit tissue using Voronoi tessellations. Comput. Electron. Agric. 52, 36-48.

Nguyen, T.A.; Dresselaers, T.; Verboven, P.; D’hallewin, G.; Culledu, N.; Van Hecke P.; Nicolaï, B. M. (2005). Finite element modelling and MRI validation of 3D transient water profiles in pears during postharvest storage. J. Sci. Food and Agric. 86 (5), 745-756.

Nguyen, T.A.; Verboven, P.; Scheerlinck, N.; Vandewalle, S.; Nicolaï, B.M. (2004). Estimation of effective diffusivity of pear tissue cuticle by means of a numerical water diffusion model. J. Food Eng. 72, 63-72.

Nygards, M.; Gudmundson, P. (2002). Micromechanical modeling of ferritic/ pearlitic steels. Material Science and Eng. 325, 435-443.

Odland, J. (1988). Spatial autocorrelation. Scientific geography series. Sage publication, USA.

Roudot, A. C.; Duprat, F.; Pietri, E. (1990). Simulation of a penetrometric test on apples using Voronoi-Delaunay tessellation. Food Structure. 9, 215-222.

Schabenberger, O.; Pierce, F. (2002). Contemporary statistical models. CRC press, USA.

Schotsmans, W. (2003). Gas diffusion properties of pome fruit in relation to storage potential. PhD thesis, Katholieke Universiteit Leuven, Leuven, Belgium, 186 p.

Sokal, R.R; Oden, N.L; Thomson, B. A. (1998). Local spatial autocorrelation in biological variables. Biol. J. Linn. Soc. 65, 41-62.

Veraverbeke E.A.; Verboven, P.; Oostveldt, P.V.; Nicolaï, B.M. (2003). Prediction of moisture loss across the cuticle of apple (Malus sylvestris subsp. mitis (Wallr.)) during storage: Part 1. Model development and determination of diffusion coefficients. Postharvest Biol. Tec. 30, 75-88.

Veraverbeke E.A.; Verboven, P.; Oostveldt, P.V.; Nicolaï, B.M. (2003). Prediction of moisture loss across the cuticle of apple (Malus sylvestris subsp. mitis (Wallr.)) during storage: Part 2. Model simulation and practical applications. Postharvest Biol. Tec.30, 89-97. 